Supporting Information:

\title{
Change of Initial Yield of Hydrated Electron with Uridine Monophosphate Concentration Is Related to the Excitation Photon Energy in Transient
}

\section{Absorption Spectroscopy}

Songqiu Yanga*, Yan Zhang ${ }^{\mathrm{b}}$, Xi Zhao ${ }^{\mathrm{a}}$

a State Key Laboratory of Molecular Reaction Dynamics, Dalian Institute of Chemical Physics, Chinese Academy of Sciences, Zhongshan Road 457, Dalian 116023, China.

b Institute of Molecular Sciences and Engineering, Shandong University, Qingdao, Binhai Road 72, Qingdao 266237, China.

*Correspond Author: Songqiu Yang, sqyang@dicp.ac.cn

\section{Contents:}

Figure S1. Transient absorption spectra of 0.1 M UMP solution, $260 \mathrm{~nm}$ pump.

Figure S2. Transient absorption spectra of 0.1 M UMP solution, $320 \mathrm{~nm}$ pump.

Figure S3. Normalized evolved spectra of pure water, $0.1 \mathrm{M}$ UMP and $0.5 \mathrm{M}$ UMP solutions at 5 ps. $320 \mathrm{~nm}$ pump.

Figure S4. Fitted the $\mathrm{e}_{\mathrm{aq}}{ }^{-}$decay lines by convolution of a Gaussian instrument response function with the geminate recombination function. ${ }^{1,2}$ The $\mathrm{e}_{\mathrm{aq}}{ }^{-}$survival probability is described as following: $\mathrm{P}(\mathrm{r}, \mathrm{t})=\int_{r>R}^{\infty}\left(1-\frac{R}{r}\right) \operatorname{erfc}\left(\frac{r-R}{2 \sqrt{D t}}\right) \exp \left(-\frac{r^{2}}{2 \sigma^{2}}\right) \mathrm{dr}$. The diffusion coefficients of $\mathrm{e}_{\mathrm{aq}}$, $\mathrm{OH}$ radical and $\mathrm{HPO}_{4}{ }^{2-}$ in water are $4.5 \times 10^{-9} \mathrm{~m} \mathrm{~s}^{-1}, 2.8 \times 10^{-9} \mathrm{~m} \mathrm{~s}^{-1}$ and $\sim 1 \times 10^{-9} \mathrm{~m} \mathrm{~s}^{-1}$. The fitted $\mathrm{e}_{\mathrm{aq}}{ }^{-}$escape probabilities are 0.50 for pure water at $260 \mathrm{~nm}$ excitation (red hollow square), 0.30 for pure water at $320 \mathrm{~nm}$ excitation (green triangle) and 0.38 for $\mathrm{Na}_{2} \mathrm{HPO}_{4}$ solution at $320 \mathrm{~nm}$ excitation (blue hollow circle).

Figure S5. Curve of the transmitted light intensity and the incident light intensity of the pure water sample. $260 \mathrm{~nm}$ pump. The blue Fit Curve 1 is obtained by fitting with the Equation S1, where $\mathrm{k}=1$, $\mathrm{C}=0$; and the red Fit Curve 2 is obtained by adding a constant after the Equation $\mathrm{S} 1$, where $\mathrm{k}=1, \mathrm{C}=-$ 8.9; the green Fit Curve 3 is obtained by fitting with the Equation $\mathrm{S} 1$, where $\mathrm{k}=0.79, \mathrm{C}=0$.

Figure S6. Saturable absorption absorbance was consistent for $0.05 \mathrm{M}, 0.1 \mathrm{M}$ and $0.5 \mathrm{M}$ UMP solutions

\section{Equation S1}




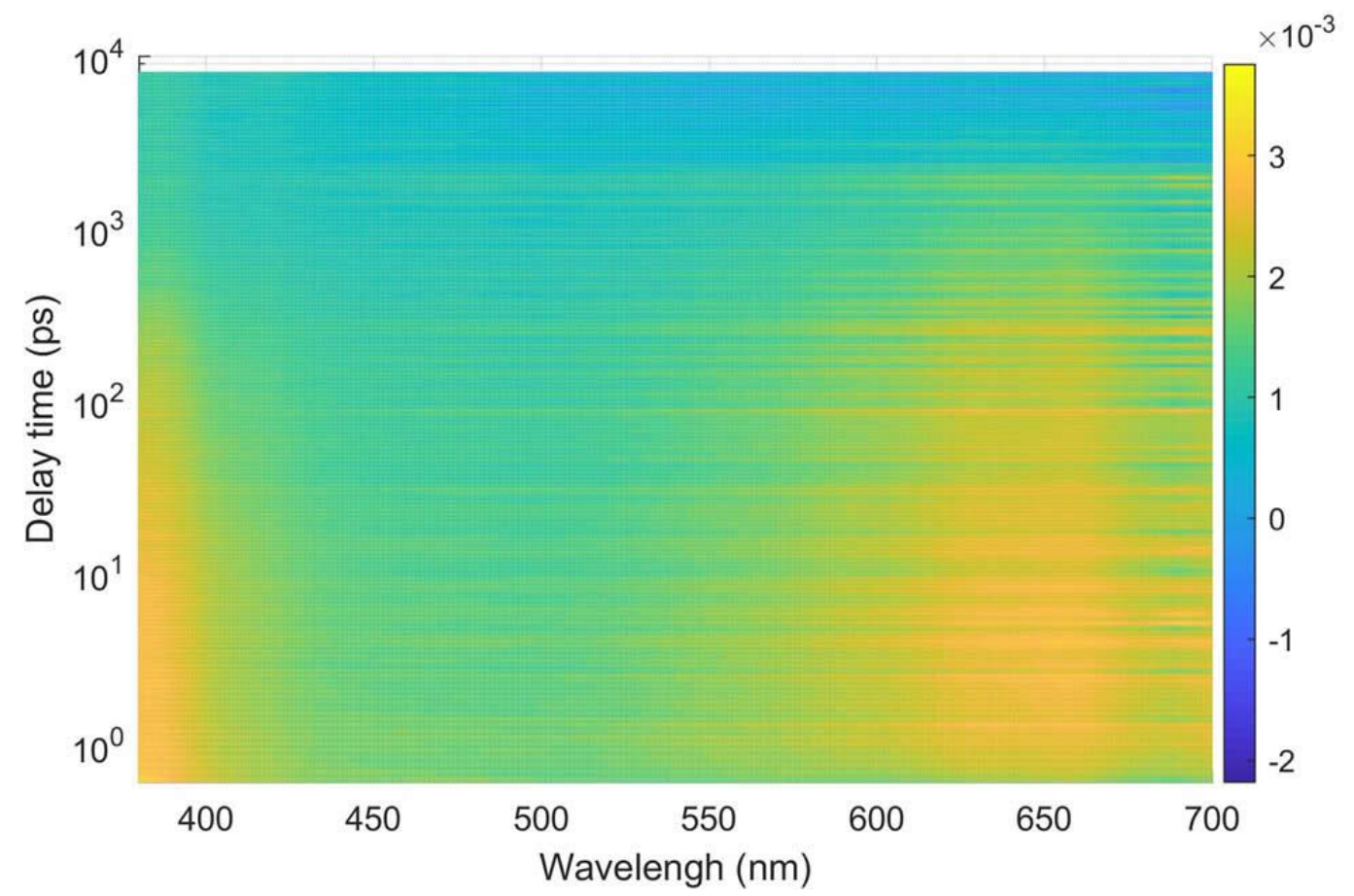

Figure S1. Transient absorption spectra of 0.1 M UMP solution, $260 \mathrm{~nm}$ pump. 


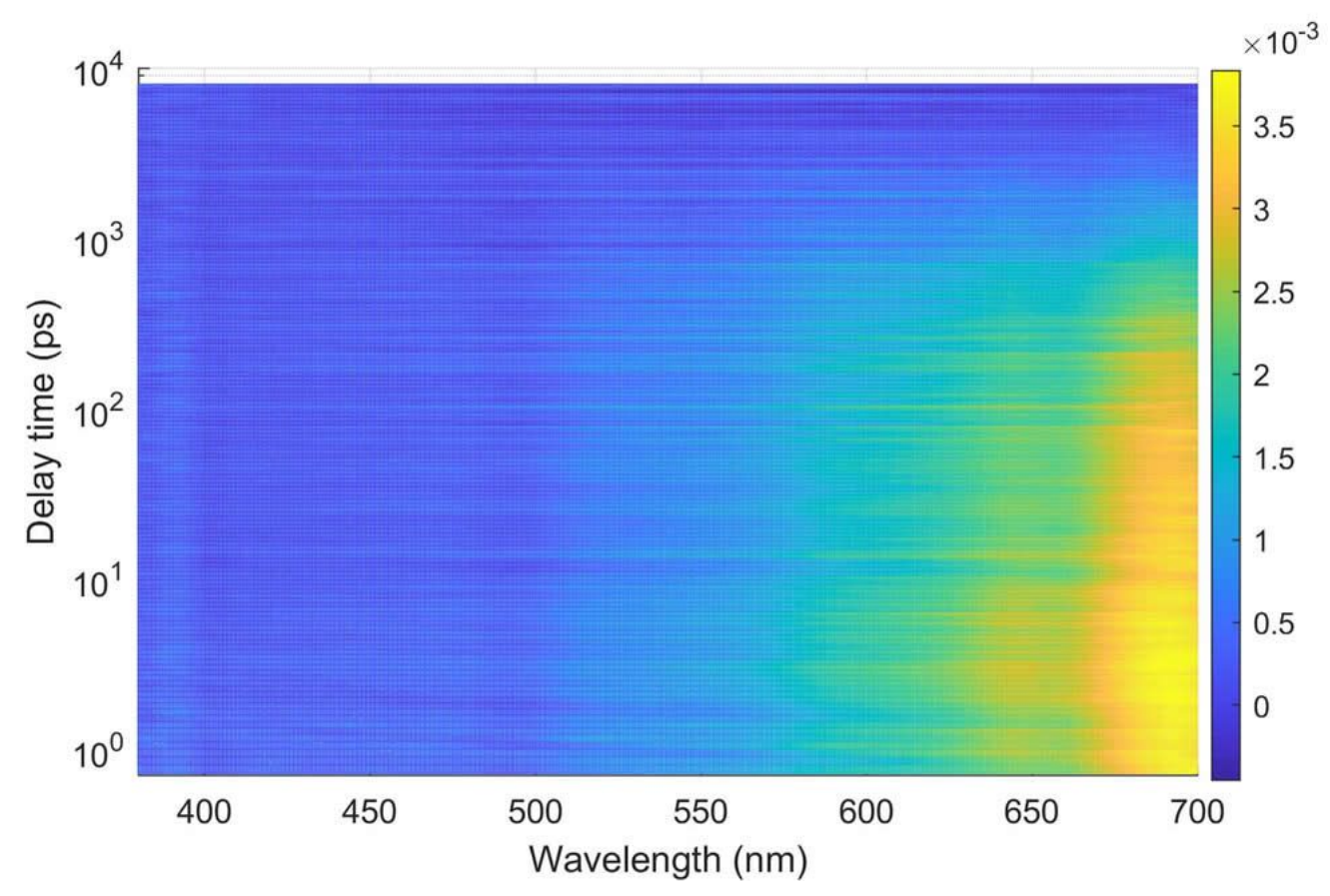

Figure S2. The transient absorption spectra of 0.1 M UMP solution, $320 \mathrm{~nm}$ pump. 


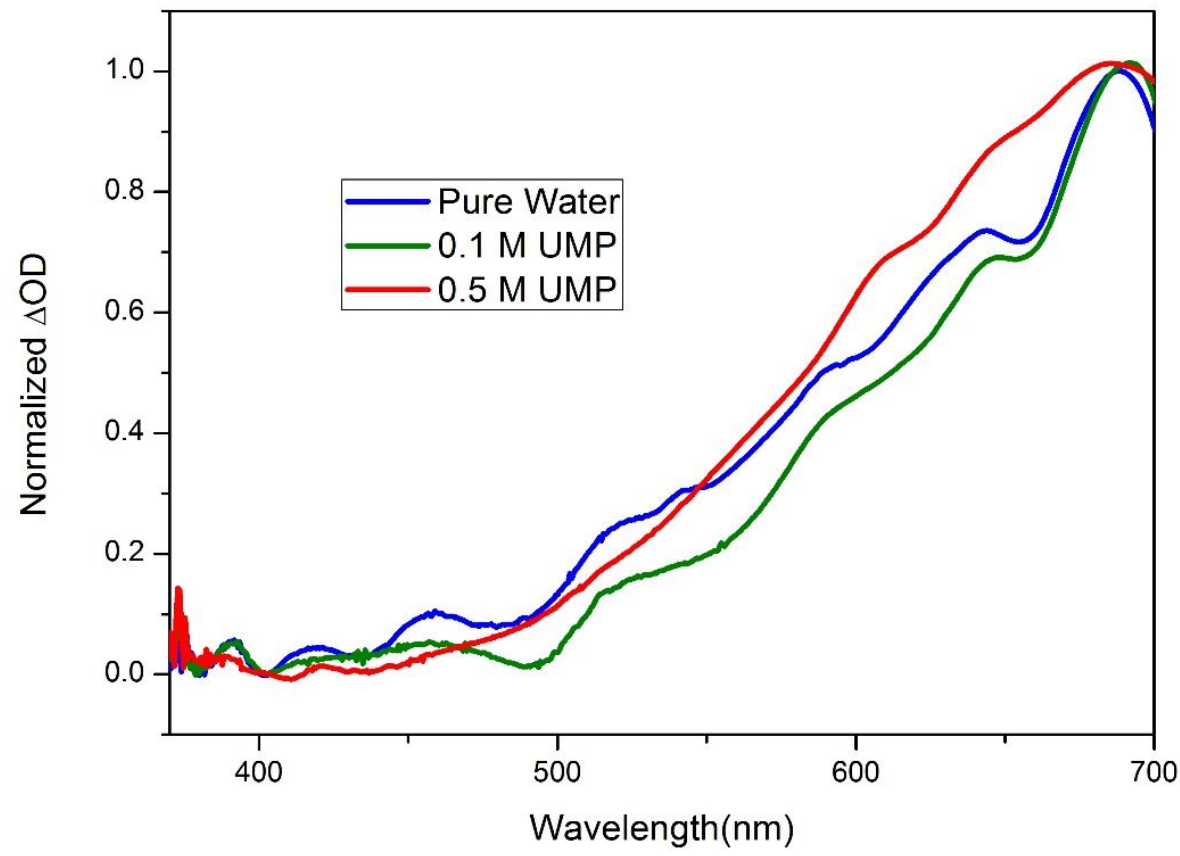

Figure S3. Normalized evolved spectra of pure water, $0.1 \mathrm{M}$ UMP and $0.5 \mathrm{M}$ UMP solutions at 5 ps. $320 \mathrm{~nm}$ pump. 


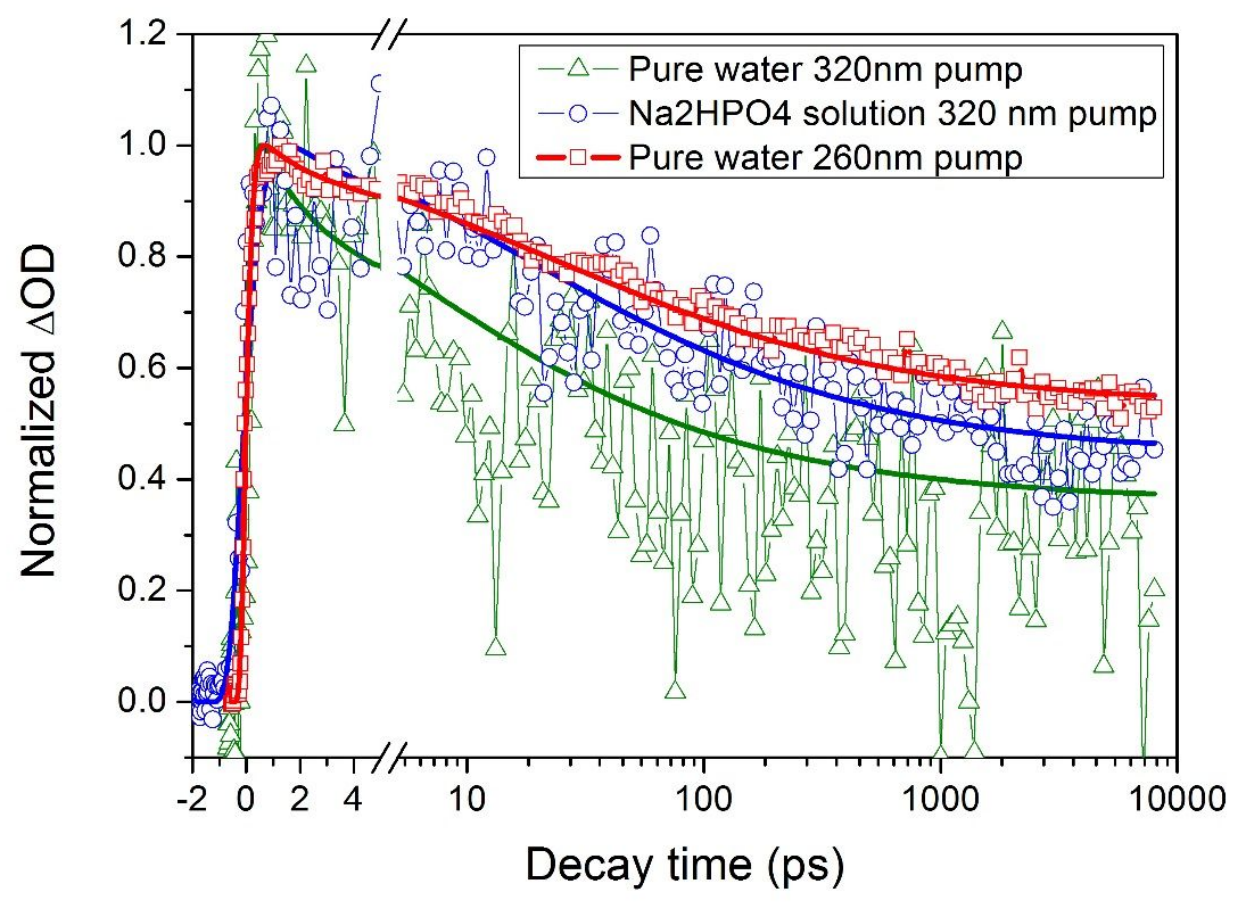

Figure S4. Fitted the $\mathrm{e}_{\mathrm{aq}}{ }^{-}$decay lines by convolution of a Gaussian instrument response function with the survival probability function. ${ }^{1,2}$ The $\mathrm{e}_{\mathrm{aq}}{ }^{-}$survival probability is described as following: $\mathrm{P}$ $(\mathrm{r}, \mathrm{t})=\int_{r>R}^{\infty}\left(1-\frac{R}{r}\right) \operatorname{erfc}\left(\frac{r-R}{2 \sqrt{D t}}\right) \exp \left(-\frac{r^{2}}{2 \sigma^{2}}\right) \mathrm{dr}$. The diffusion coefficients of $\mathrm{e}_{\mathrm{aq}}{ }^{-}, \mathrm{OH}$ radical and $\mathrm{HPO}_{4}{ }^{2-}$ in water are $4.5 \times 10^{-9} \mathrm{~m} \mathrm{~s}^{-1}, 2.8 \times 10^{-9} \mathrm{~m} \mathrm{~s}^{-1}$ and $\sim 1 \times 10^{-9} \mathrm{~m} \mathrm{~s}^{-1}$. The fitted e eaq escape probabilities are 0.50 for pure water at $260 \mathrm{~nm}$ excitation (red hollow square), 0.30 for pure water at $320 \mathrm{~nm}$ excitation (green triangle) and 0.38 for $\mathrm{Na}_{2} \mathrm{HPO}_{4}$ solution at $320 \mathrm{~nm}$ excitation (blue hollow circle). 


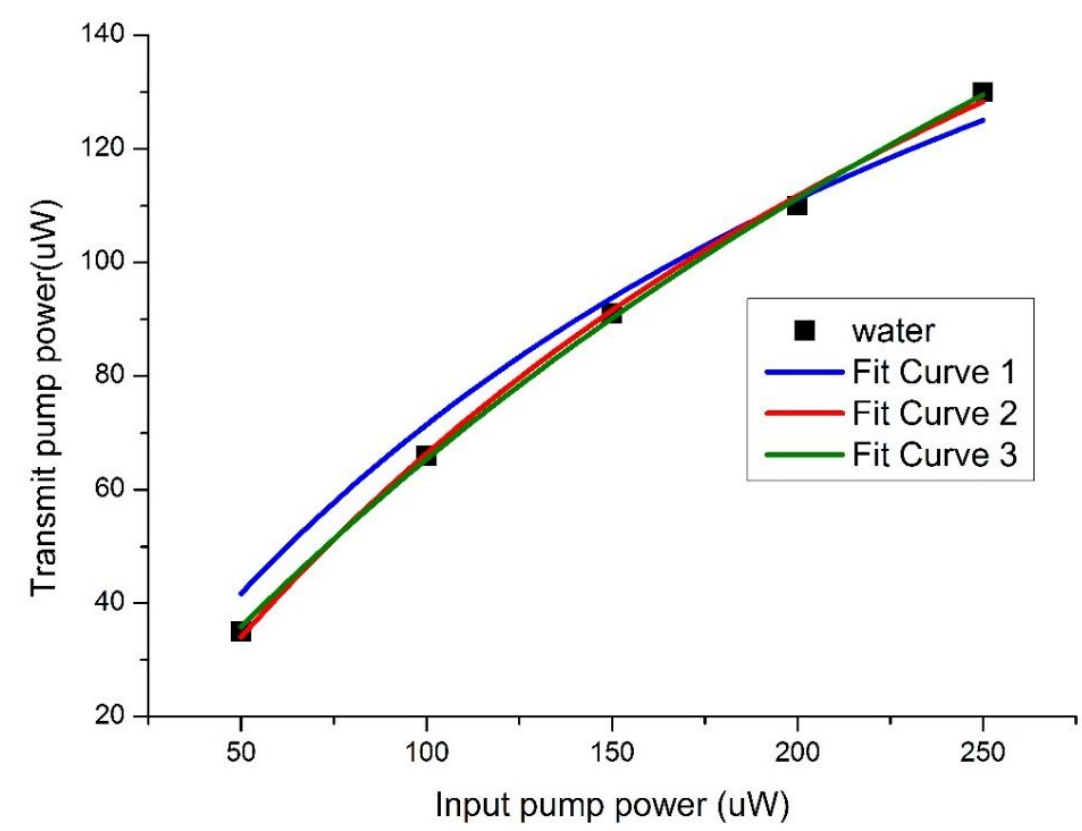

Figure S5. Curve of the transmitted light intensity and the incident light intensity of the pure water sample. 260nm pump. The blue Fit Curve 1 is obtained by fitting with the Equation S1, where k=1, $\mathrm{C}=0$; and the red Fit Curve 2 is obtained by adding a constant after the Equation $\mathrm{S} 1$, where $\mathrm{k}=1, \mathrm{C}=-$ 8.9; the green Fit Curve 3 is obtained by fitting with the Equation $\mathrm{S} 1$, where $\mathrm{k}=0.79, \mathrm{C}=0$. 


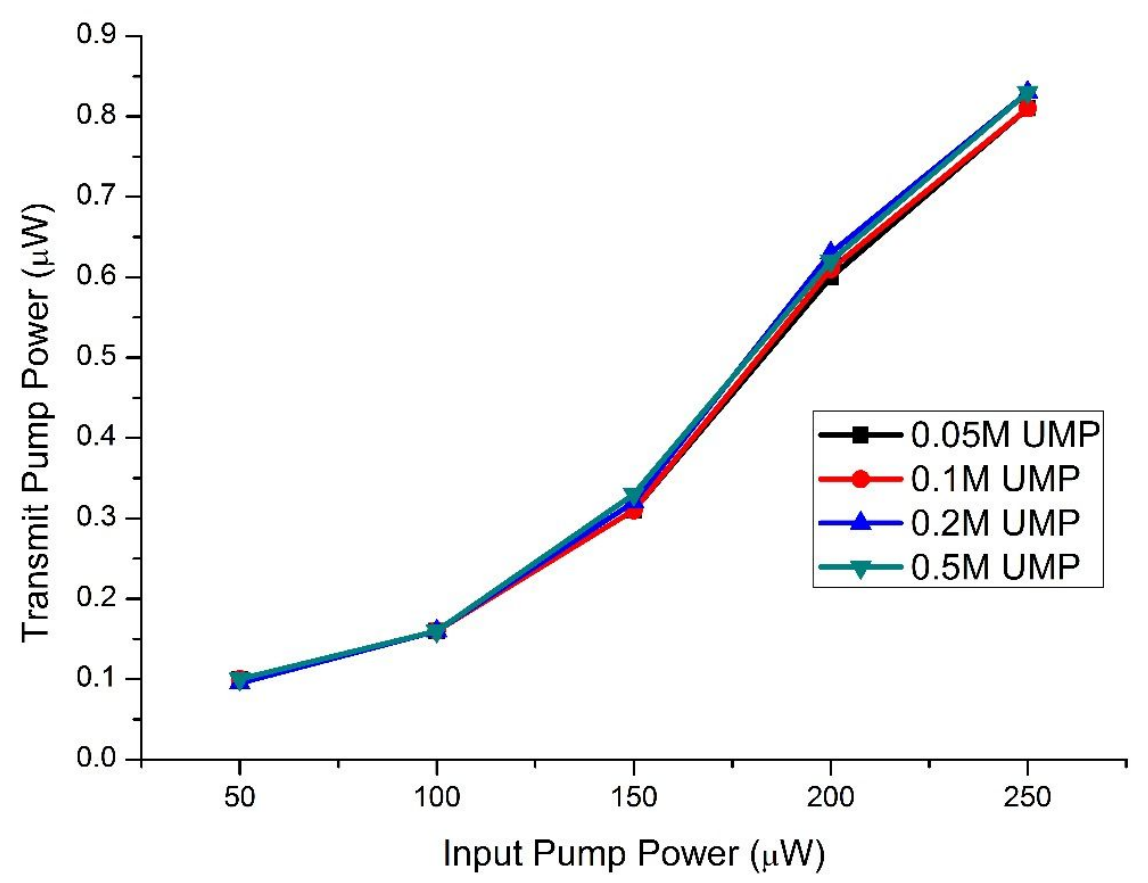

Figure S6. Transmittance pump power versus input pump power in varies UMP concentration solutions. The transmittances are consistent for $0.05 \mathrm{M}, 0.1 \mathrm{M}$ and $0.5 \mathrm{M}$ UMP solutions.

$\mathrm{I}(\lambda)=\frac{k I_{0}(\lambda)}{1+\beta(\lambda) k I_{0}(\lambda)}+C \quad$ (Equation $\left.\mathrm{S} 1\right)$

$\mathrm{I}(\lambda)$ is the transmittance pump power, $\mathrm{I}_{0}(\lambda)$ is the incident pump power, $\mathrm{k}$ is a reflector constant, and $\beta(\lambda)$ is the two-photon absorption extinction coefficient.

\section{References}

1. Pimblott, S. M., Independent pairs modeling of the kinetics following the photoionization of liquid water. J. Phys. Chem. 1991, 95 (18), 6946-6951.

2. Crowell, R. A.; Bartels, D. M., Multiphoton ionization of liquid water with 3.0-5.0 eV photons. J. Phys. Chem. 1996, 100 (45), 17940-17949. 